Original Article

\title{
Determining the Factors Predicting the Response to Anti- HER2 Therapy in HER2-Positive Breast Cancer Patients
}

\author{
Ji Young You $\mathrm{MD}^{1}$, Kyoung Hwa Park $\mathrm{MD}^{2}$, Eun Sook Lee $\mathrm{MD}^{3}$, Youngmee Kwon $\mathrm{MD}^{3}$, Kyoung Tae Kim \\ $\mathrm{PhD}^{4}$, Seungyoon Nam $\mathrm{PhD}^{5}$, Donghee Kim $\mathrm{MD}^{6}$, Jeoung Won Bae MD${ }^{1}$ \\ ${ }^{1}$ Division of Breast and Endocrine, Department of Surgery, Korea University Medical Center, Seoul, Korea \\ ${ }^{2}$ Department of Medical Oncology, Korea University Medical Center, Seoul, Korea \\ ${ }^{3}$ Center for Breast Cancer, National Cancer Center, Research Institute \& Hospital, Goyang, Gyeonggi, Korea \\ ${ }^{4}$ Department of Molecular Dynamic Research, National Cancer Center, Research Institute and Hospital, Goyang, \\ Gyeonggi, Korea \\ ${ }^{5}$ Department of Genome Medicine and Science, Gachon University Gil Medical Center, Incheon, Korea \\ ${ }^{6}$ Department of Surgery, Eulji General Hospital, Eulji University School of Medicine, Seoul, Korea \\ *Correspondence: Jeoung Won Bae \\ c \\ Goryeodae-ro 73, Seongbuk-gu, Seoul 02841, Republic of Korea \\ Phone: $+82-2-920-6770$ \\ E-mail: kujwbae@korea.ac.kr
}

Received: date; Accepted: date; Published: date

\begin{abstract}
Background: We aimed to identify overexpressed genes or related pathways associated with good responses to anti-HER2 therapy and to suggest a model for predicting drug response in neoadjuvant therapy with trastuzumab in HER2-positive breast cancer patients. Methods: We recruited 64 women with breast cancer and categorized them into three groups according to anti-HER2 therapy response. RNA from twenty core needle biopsy paraffin-embedded tissues and four cultured cell was extracted, reverse transcribed, and subjected to microarray. The obtained data were analyzed using Gene Ontology, Kyoto Encyclopedia of Genes and Genomes, and database for annotation, visualization, and integrated discovery. Results: In total, 6,656 genes differentially expressed between trastuzumab-susceptible and trastuzumab-resistant cell lines (3,224 upregulated and 3,432 downregulated). Expression changes in 34 genes in several pathways were found to be related to the response to trastuzumab-containing treatment, interfering with adhesion to other cells or tissues (focal adhesion) and regulating extracellular matrix interactions and phagosome action. Thus, decreased tumor invasiveness and enhanced drug effects might be the mechanisms explaining the better drug response in complete response group. Conclusions: This multigene assay-based study provides insights into breast cancer signal transduction and the
\end{abstract}


possible prediction of treatment response to targeted therapies such as trastuzumab.

Keywords: HER2; neoadjuvant; pathologic complete response; trastuzumab

\section{Introduction}

Breast cancer is one of the most common malignancies among women worldwide and is considered a heterogeneous disease. As determined by immunohistochemistry (IHC), it is categorized into several subtypes according to receptor status, and different treatments are administered for the different subtypes. Overexpression of human epithelial growth factor receptor 2 (HER2) occurs in $25-30 \%$ of all breast cancers and is associated with poor prognosis.[1] HER2 activation occurs by dimerization with other proteins in the family, and multiple downstream pathways are required for the abnormal proliferation of cancer cells.[2]

Trastuzumab is a recombinant humanized monoclonal antibody directed against the extracellular domain of the HER2 protein.[3] Trastuzumab binds to HER2 and reduces the activity of downstream signaling pathways, including the phosphatidylinositol 3-kinase (PI3K) and mitogen-activated protein kinase (MAPK) cascades, thereby increasing p27Kip1 levels and promoting cell cycle arrest and apoptosis.[4] In patients with early-stage operable and metastatic HER2-positive breast cancer, trastuzumab has well-established survival benefits when included in the chemotherapy regimen.

Pathologic complete response (pCR) is defined as the absence of residual invasive cancer in the breast and lymph nodes in surgical specimens after neoadjuvant therapy. In the neoadjuvant setting, compared with chemotherapy without trastuzumab, chemotherapy with trastuzumab significantly improves pCR rates and reduces the risks of relapse, disease progression, and death.[5] pCR is considered an important prognostic factor and is associated with long-term survival. Thus, pCR has been adopted as the primary end point for neoadjuvant trials, and it reaches up to $78 \%$ in patients treated with trastuzumab.[6,7] In the clinical course, low pCR rates for anti-HER2 treatment are observed in some patient groups, suggesting the variation in HER2 expression status, i.e., intratumoral HER2 heterogeneity.[8] It is important to select suitable patients for neoadjuvant antiHER2 therapy owing to the limited opportunity for treatment before surgery. However, the mechanism underlying the therapeutic effect and the factors contributing to treatment response have not been established, and a consensus regarding the criteria for patient screening is lacking.

The aim of this study was to identify overexpressed genes or related pathways associated with good responses to

anti-

HER2 therapy and to suggest a model for predicting drug response in neoadjuvant systemic therapy with trast uzumab in HER2-positive breast cancer patients.

\section{Materials and Methods}

\section{Patients}

Patients consented to the use of the tissue specimens for research purposes, as approved by the Institutional 
Review Board (IRB) of the National Cancer Center (Goyang, Gyeonggi-do, Korea) (NCC2015-0025). Inclusion criteria were as follows: locally advanced breast cancer without distant metastasis, HER2-positive status on IHC or silver-enhanced in situ hybridization, or prior surgical resection with neoadjuvant anti-HER2 therapy with chemotherapy (regimen: adriamycin and cyclophosphamide for four cycles, followed by taxane and trastuzumab for four cycles). We excluded the patients who had undergone chemotherapy previously or had a history of radiotherapy, of previous malignancy of another organ, or of discontinuing treatment due to any reason. We identified 64 consecutive women satisfying the inclusion criteria at our institution between July 2005 and January 2014.

These patients were categorized into groups according to drug response as per the RECIST criteria (version 1.1): complete response (CR), partial response (PR), stable disease (SD), and progressive disease (PD). However, only three patients with SD and one with PD were detected; hence, owing to the small number, they were categorized into a drug resistance (DR) group. Finally, the patients were classified into three groups. The CR, PR, and DR groups included 25 (39.1\%), 35 (54.6\%), and 4 (6.3\%) cases, respectively. Among these, twenty patients were finally enrolled (eleven CR patients and nine PR patients), from whom core needle biopsy (CNB) tissues obtained at the initial diagnosis were available as paraffin-embedded blocks. An outline of the patient selection process is given in Figure 1.

We collected patients' clinicopathological information, such as patient and tumor characteristics, surgical methods, adjuvant therapy, and response to treatment by reviewing our institution database.

\section{Study design}

In the above patient pool, we performed experiments using pre-chemotherapy paraffin-embedded CNB specimens. Of the available twenty initial paraffin-embedded CNB specimens, eleven belonged to the CR group and nine to the PR group. Unfortunately, the DR group could not be tested because not all the patients underwent biopsy at our institution. For validation, in vitro experiments were conducted similarly. Trastuzumabsusceptible cell lines and trastuzumab-resistant cell lines were used in the study.

\section{Experimental methods}

Cell lines, reagents, and cell culture

Parent cell lines and resistant cell lines of SKBR3 and BT474 from the American Type Culture Collection (ATCC, Manassas, VA, USA) and the German Collection of Microorganisms and Cell Cultures (DSMZ, Braunschweig, Germany) were donated by Dr. Park, Professor of the Department of Medical Oncology, Korea University. Resistant cells were developed by culturing the SKBR3 and BT474 epithelial breast cancer cells in the presence of $50 \mu \mathrm{g}$ trastuzumab (Roche, Basel, Switzerland) for around three years. Parent cells were cultured in parallel to the resistant ones without the addition of trastuzumab. Resistance was verified by cell viability assays. Trastuzumab markedly reduced the growth of wild-type parent cells compared to trastuzumab-resistant cells.[9] 
SKBR3 cells were grown in Roswell Park Memorial Institute medium 1640 medium (Sigma-Aldrich, St. Louis, MO, USA), and BT474 cells were grown in Dulbecco's modified Eagle's medium (Hyclone, Logan, UT, USA) with $10 \%$ fetal bovine serum (Hyclone) at $37^{\circ} \mathrm{C}$ in $5 \% \mathrm{CO}_{2 \cdot}[10]$

\section{RNA extraction and cDNA synthesis}

RNA extraction was performed using the $\operatorname{mirVana}^{\mathrm{TM}}$ isolation kit (Ambion ${ }^{\circledR}$, Thermo Fisher Scientific, Waltham, MA, USA) on each sample (20 CNB paraffin-embedded tissues and 4 cultured cell lines). cDNA synthesis was performed by reverse transcription from extracted RNA as follows. First, $2 \mu \mathrm{L}$ random primer or oligo dT was added to $10 \mu \mathrm{L}$ extracted RNA, heated at $70^{\circ} \mathrm{C}$ for $10 \mathrm{~min}$, and cooled for $5 \mathrm{~min}$ at $45^{\circ} \mathrm{C}$. Then, $8 \mu \mathrm{L}$ of the mix solution $(4 \mu \mathrm{L} 5 \times$ buffer, $1 \mu \mathrm{L}$ DL-dithiothreitol, $1 \mu \mathrm{L} 10 \times$ deoxyribonucleotide triphosphates, $0.5 \mu \mathrm{L}$ Superscript reverse transcriptase, $0.5 \mu \mathrm{L}$ RNase inhibitor, and $1 \mu \mathrm{L}$ DNase/RNase-free water) were added to each sample. The mixture was incubated overnight at $45^{\circ} \mathrm{C}$ and finally at $70^{\circ} \mathrm{C}$ for $10 \mathrm{~min}$, and stored at $-20^{\circ} \mathrm{C}$. Purity quality control was performed to determine the feasibility of the experiment.

\section{GeneChip microarray}

The DNA microarray approach, in which specific gene sequences are spotted on a glass plate, is a useful approach for large-scale expression analyses. Briefly, extracted RNA is reverse transcribed to obtain cDNA, labeled with different fluorescent materials for each sample, and spotted. It is a technique used to determine the degree of expression of each gene by measuring the intensity of emitted light by analyzing the amount of cDNA bound to each spot by injecting a laser with an optical scanner.

The Affymetrix (Santa Clara, CA, USA) whole-transcript (WT) expression array process was executed according to the manufacturer's protocol (GeneChip Whole Transcript PLUS reagent kit). cDNA was synthesized using the GeneChip WT amplification kit as described by the manufacturer. The sense cDNA was then fragmented and biotin-labeled with terminal deoxynucleotidyl transferase using the GeneChip WT terminal labeling kit. Approximately $5.5 \mu$ g labeled DNA target was hybridized to the Affymetrix GeneChip ${ }^{\circledR}$ Human 2.0 ST Array at $45^{\circ} \mathrm{C}$ for $16 \mathrm{~h}$. Hybridized arrays were washed and stained on a GeneChip Fluidics Station 450 and scanned on a GCS3000 Scanner (Affymetrix).

\section{Data analysis}

Various analysis tools were used owing to the extensive amount of GeneChip data. Analyses of biological net works are essential to study functional genomic data. A series of analysis steps were repeated with and withou $\mathrm{t}$ differentially expressed genes (DEGs) to determine the false positive rate. Gene enrichment and functional a nnotation analysis for a significant probe list was performed using Gene Ontology (GO) and the Kyoto encycl opedia of genes and genomes (KEGG). All data analysis and visualization of DEGs was conducted using the $\mathrm{R}$ project for statistical computing (version 3.1.2). The functional annotation toolthe database for annotation, visualization, and integrated discovery (DAVID) can organize and condense large gene lists into biologically meaningful modules. It changes functional annota 
tion analysis from term- or gene-centric to biological modulecentric.[11] It was used to confirm the expression level, fold change, and pvalue of each gene. Significant genes identified in the microarray were also analyzed by DAVID for functiona 1 annotation, and results were visualized by constructing a Venn diagram. The differences were considered sig nificant when the $p$-value was less than 0.05 .

\section{Results}

\section{Patient demographics}

Twenty patients were enrolled in this study. According to the IRB policy, the appropriate tissue from patients fulfilling our study's inclusion criteria were provided with detailed personal information masked, and were divided into several groups according to the patient's treatment response. Clinical information related to the experimental group indicated that age, receptor status, regimen used, and operative method were distributed evenly among the groups. The study flow is outlined in Figure 1, as mentioned in the methods section, and the clinicopathological data are summarized in Table 1.

\begin{tabular}{|c|c|c|c|}
\hline \multicolumn{4}{|c|}{ From July 2005 to January 2014, at the Center of Breast Cancer, National Cancer Center } \\
\hline \multicolumn{2}{|c|}{$\begin{array}{l}\text { Inclusion Criteria } \\
\text { 1. Locally advanced breast cancer without distant metastasis } \\
\text { 2. C-erbB2 } 3 \text { positive in IHC stain or SISH positive } \\
\text { 3. Who underwent neoadjuvant anti HER2 therapy } \\
\text { (AC*4 cycles and } \mathrm{T}^{\star}+\text { trastuzumab } 4 \text { cycles) } \\
{ }^{*} \mathrm{AC}: \text { Adriamycin + cyclophosphamide, T: anthracyclin } \\
\text { 4. Who underwent surgical resection }\end{array}$} & \multicolumn{2}{|r|}{$\begin{array}{l}\text { Exclusion Criteria } \\
\text { 1. Previous chemotherapy or radiation history } \\
\text { 2. Previous malignancy of other organ } \\
\text { 3. Quit treatment due to any reason } \\
\text { 4. Without informed consents }\end{array}$} \\
\hline \multicolumn{4}{|c|}{$\begin{array}{l}\text { : Total } 64 \text { patients enrolled } \\
\text { Categorized into } 3 \text { groups by responses to treatment using RECIST criteria v.1.1 }\end{array}$} \\
\hline $\begin{array}{l}\text { Complete response : } \\
25 / 64(39.1 \%)\end{array}$ & \multicolumn{2}{|c|}{$\begin{array}{c}\text { Partial response : } \\
35 / 64(54.6 \%)\end{array}$} & $\begin{array}{l}\text { Drug resistance : } \\
\text { (non-refractory) } \\
4 / 64(6.3 \%)\end{array}$ \\
\hline \multicolumn{4}{|c|}{$\begin{array}{l}: 20 \text { available diagnostic core needle biopsy paraffin embedded tissue before neoadjuvant therapy } \\
\text { (CR } 11 \text { vs. PR 9) }\end{array}$} \\
\hline
\end{tabular}

Figure 1. Patients selection flow for study 
Table 1. Clinicopathological status of the enrolled patients

\begin{tabular}{|c|c|c|c|}
\hline Variables & $\mathrm{CR}(\mathrm{n}=11)$ & $\mathrm{PR}(\mathrm{n}=9)$ & $p$-value \\
\hline Mean age (years) & $50.4 \pm 8.89$ & $48.7 \pm 6.48$ & 0.283 \\
\hline Sex & & & NA \\
\hline Female & $11(100.0 \%)$ & $9(100.0 \%)$ & \\
\hline Hormone receptor status & & & 0.343 \\
\hline Estrogen receptor positive & $5(45.5 \%)$ & $6(66.7 \%)$ & \\
\hline Progesterone receptor positive & $2(18.2 \%)$ & $5(55.6 \%)$ & \\
\hline HER2 expression in IHC & & & 0.074 \\
\hline $2+$ & $0(0.0 \%)$ & $3(33.3 \%)$ & \\
\hline $3+$ & $11(100.0 \%)$ & $6(66.7 \%)$ & \\
\hline SISH results & Not done & & NA \\
\hline Equivocal & & $1(33.3 \%)$ & \\
\hline Positive & & $2(66.7 \%)$ & \\
\hline Neoadjuvant therapy regimen & & & NA \\
\hline $\mathrm{AC}+\mathrm{TH}$ & $11(100.0 \%)$ & $9(100.0 \%)$ & \\
\hline Operation methods & & & 0.769 \\
\hline $\mathrm{BCS}$ & $8(72.7 \%)$ & $6(66.7 \%)$ & \\
\hline TM & $3(27.3 \%)$ & $3(33.3 \%)$ & \\
\hline \multicolumn{4}{|l|}{ Postoperative pathology in CR } \\
\hline No residual tumor & $8(72.7 \%)$ & - & NA \\
\hline Remnant DCIS & $3(27.3 \%)$ & - & \\
\hline
\end{tabular}

CR: complete response; PR: partial response

IHC: immunohistochemistry; SISH: silver in situ hybridization for HER2 gene expression

AC: adriamycin+cyclophosphamide; TH: taxane + Herceptin (trastuzumab)

BCS: breast-conserving surgery; TM: total mastectomy

DCIS: ductal carcinoma in situ

\section{GeneChip microarray quality control and analysis results}

For comparison of the two groups with different responses to the same therapy, the first experiment was performed with the tumor tissue of patients, and the second experiment was performed using cell lines with different treatment responses to trastuzumab for validation. 
In our first experiment, the hierarchical clustering, heatmap, and data control were well-distributed. Both the multidimensional scaling plots were well implemented, and correlation was normal. To determine differences in gene expression in the CR and PR groups in more detail, we performed additional analyses, dividing the CR group into 2 subgroups_-no residual tumor and remnant ductal carcinoma in situ (DCIS)_according to the final pathological report.

\section{Identification of DEGs}

With a fold change of $1.5,1,473$ genes that were differentially expressed between the CR and PR groups were identified. Among these, 481 were upregulated and 992 were downregulated. In addition, 171 DEGs were identified between the CR and PR groups with a fold change of 2.0. Among them, 38 were upregulated and 133 were downregulated. More genes were identified upon comparing the PR group and CR subgroups (no residual tumor and remnant DCIS subgroups). A total of 915 significant probes were noted after comparing CR and PR groups, 2,175 after comparing the CR group and the no residual tumor subgroup, and 278 after comparing the CR group and the remnant DCIS subgroup. These data are presented in Figure 2(a).

\section{Functional GO analysis of DEGs}

To understand the relevance of these genes, a program based on GO categories of biological process, cellular component, and molecular function was employed. Figure 3(a) and 3(b) shows the main functional GO categories of transcripts that were differentially expressed between the CR and PR groups (adjusted $p<0.05$; gene count $>2$ of the up- or downregulated genes). Altered transcription of genes in the "biological process" category included a multitude of genes serving as signaling molecules or receptors. In the "cellular component" category, genes included all parts of cell structures, and in the "molecular function" category, it included genes mainly related to binding to various molecules.

In vitro validation experiments

A total of 6,656 genes differentially expressed between trastuzumab-susceptible and trastuzumab-resistant cell lines were identified. Among these, 3,224 were upregulated and 3,432 were downregulated. A total of 4,281 significant probes were noted between trastuzumab-susceptible and trastuzumab-resistant groups with a fold change of 1.5, and 2,707 probes with a fold change of 2.0 (Figure 2(b)).

GO categories in the DEGs of the second experiment showed a trend similar to that in the first experiment. In the "biological process" category, genes were mainly related to the regulation of cellular processes. Altered transcription of genes in the "cellular component" category included a multitude of genes serving as cell structures. In the "molecular function" category, observed results most similar to those of the tumor experiment, i.e., the genes were mainly related to binding and catalytic activity (Figure 3(c) and 3(d) ). 


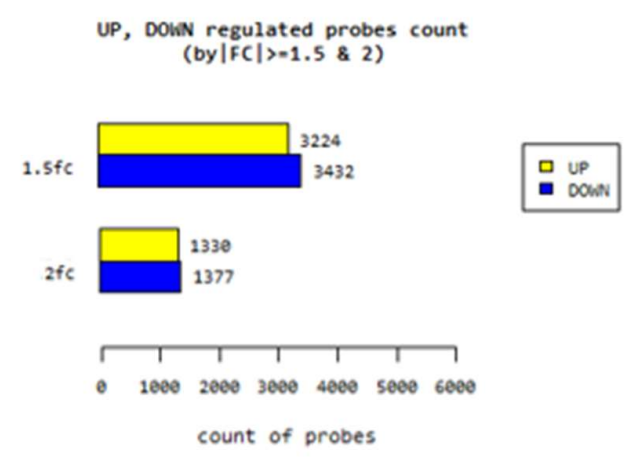

(a) DEGs in comparison between $\mathrm{CR}$ group vs. $\mathrm{PR}$ group

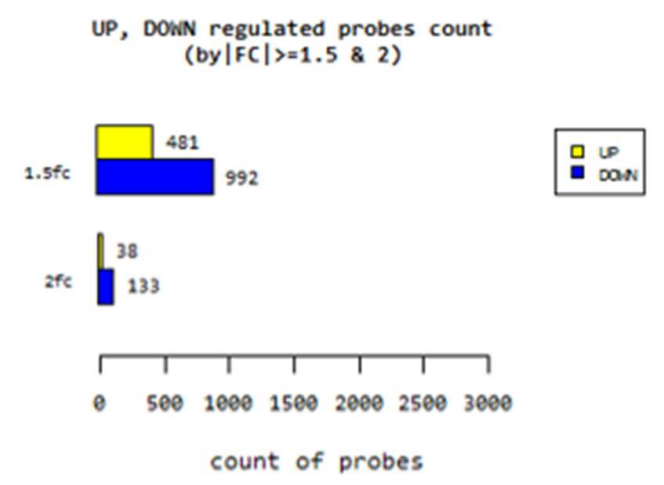

(b) DEGs in comparison between susceptible vs. resistant cell lines

Figure 2. Differentially expressed genes (DEGs) in comparison of different treatment response groups(a) and comparison of different cell line experiments(b) 
(a) GO categories in up-regulated genes
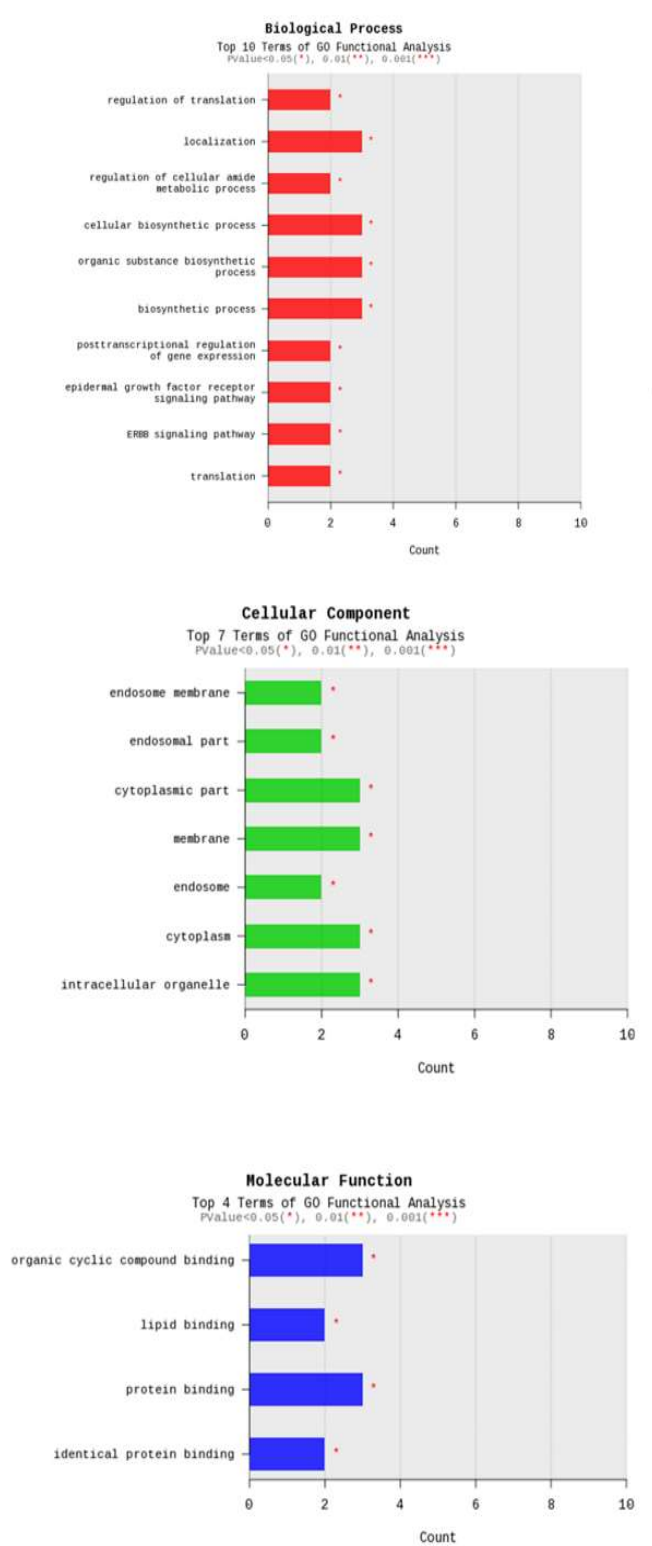

(b) GO categories in down-regulated genes
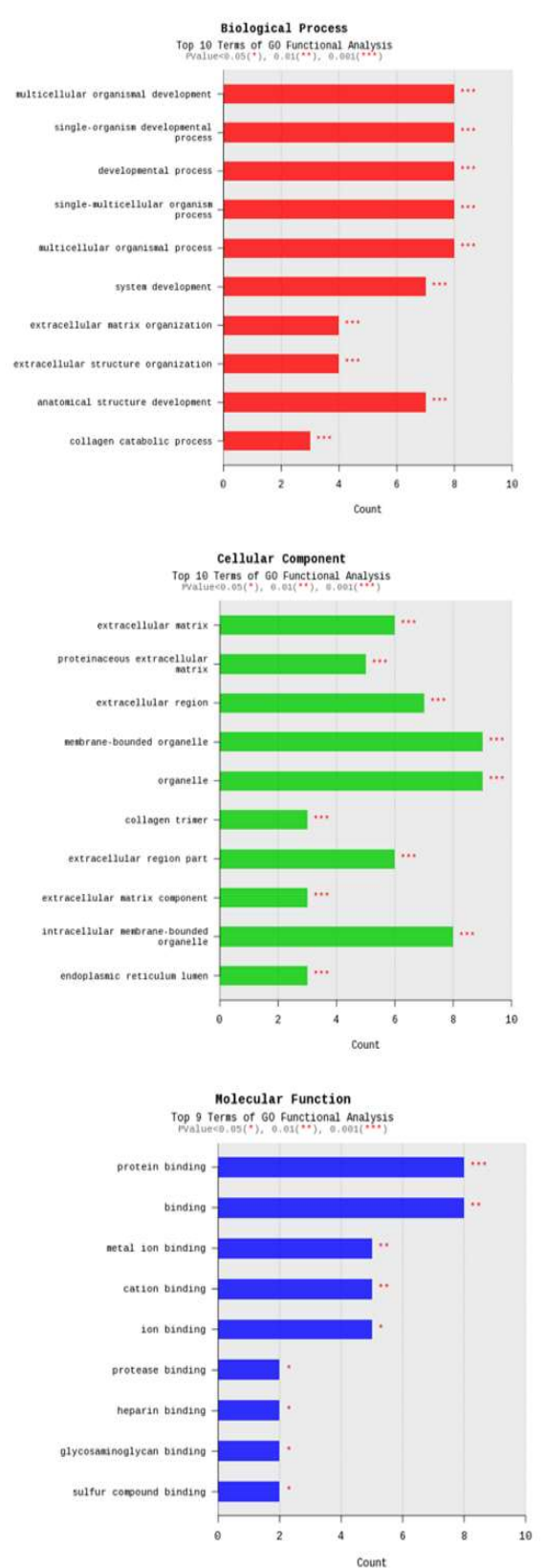
(c) GO categories in up-regulated genes
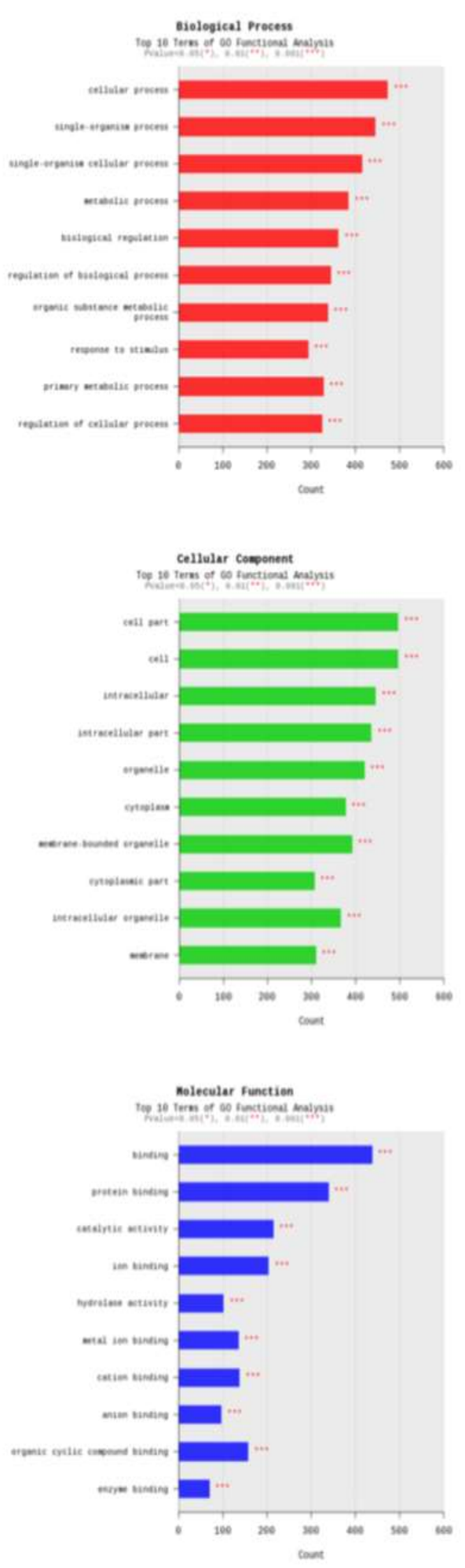

(d) GO categories in down-regulated genes
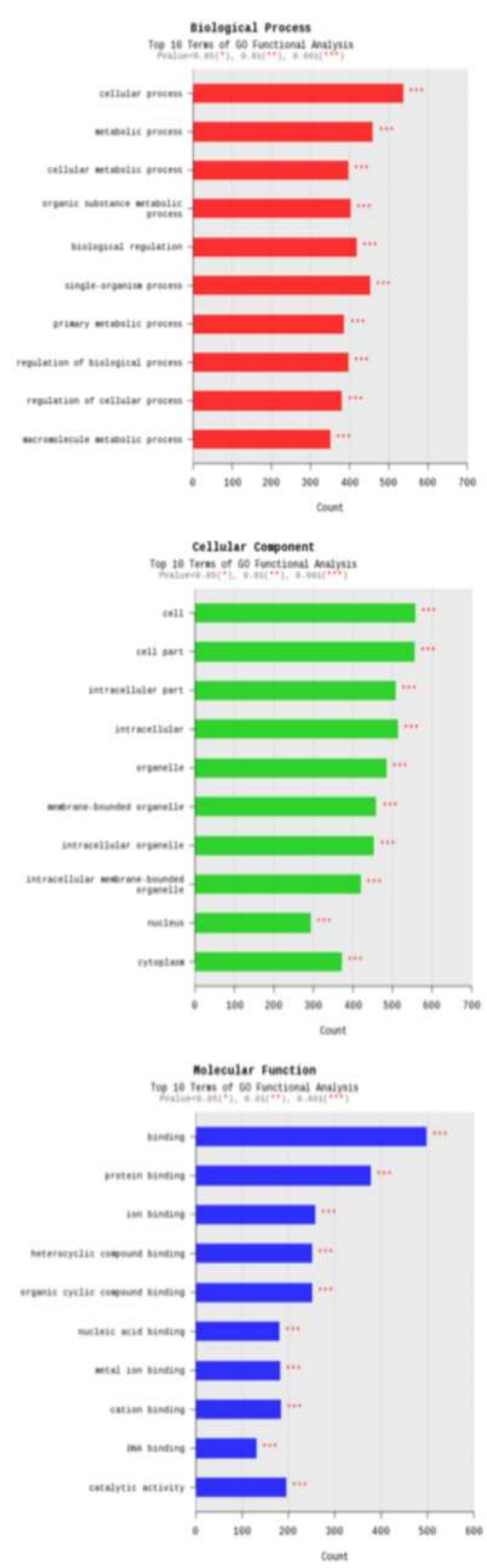

Figure 3. Gene ontology (GO) categories in differentially expressed genes of different treatment response groups(a\&b) and comparison of different cell line experiments(c\&d) 


\section{Comparison with cell lines for validation}

The genes related to functional annotation were analyzed using DAVID and visualized by a Venn diagram. A total of 34 genes were selected as the trastuzumab treatment response-related genes from the common up- and downregulated ones with various comparisons in the analysis (Figure 4).

As shown in Table 2, these 34 genes were commonly downregulated in response to trastuzumab in the CR group compared with the PR group. These genes encode a diverse group of proteins, including binding proteins (GRB7, IGFBP4), transfer domain (STARD3, XYLT2), and collagen chain (COL10A1, COL12A1, COL8A2), in association with cell signaling and other activities. Furthermore, some of these 34 genes, including ERBB2, are involved in pathways directly implicated in HER2 signaling.

Pathway analysis was performed on the 34 genes that showed different responses to trastuzumab treatment. Most of the genes were strongly correlated with 3 pathways: related to focal adhesion, extracellular matrix (ECM)-receptor interaction, and the phagosome. These pathways are linked to the PI3k pathway or MAP kinase pathway in that they have been proved to be associated with HER family pathways. 


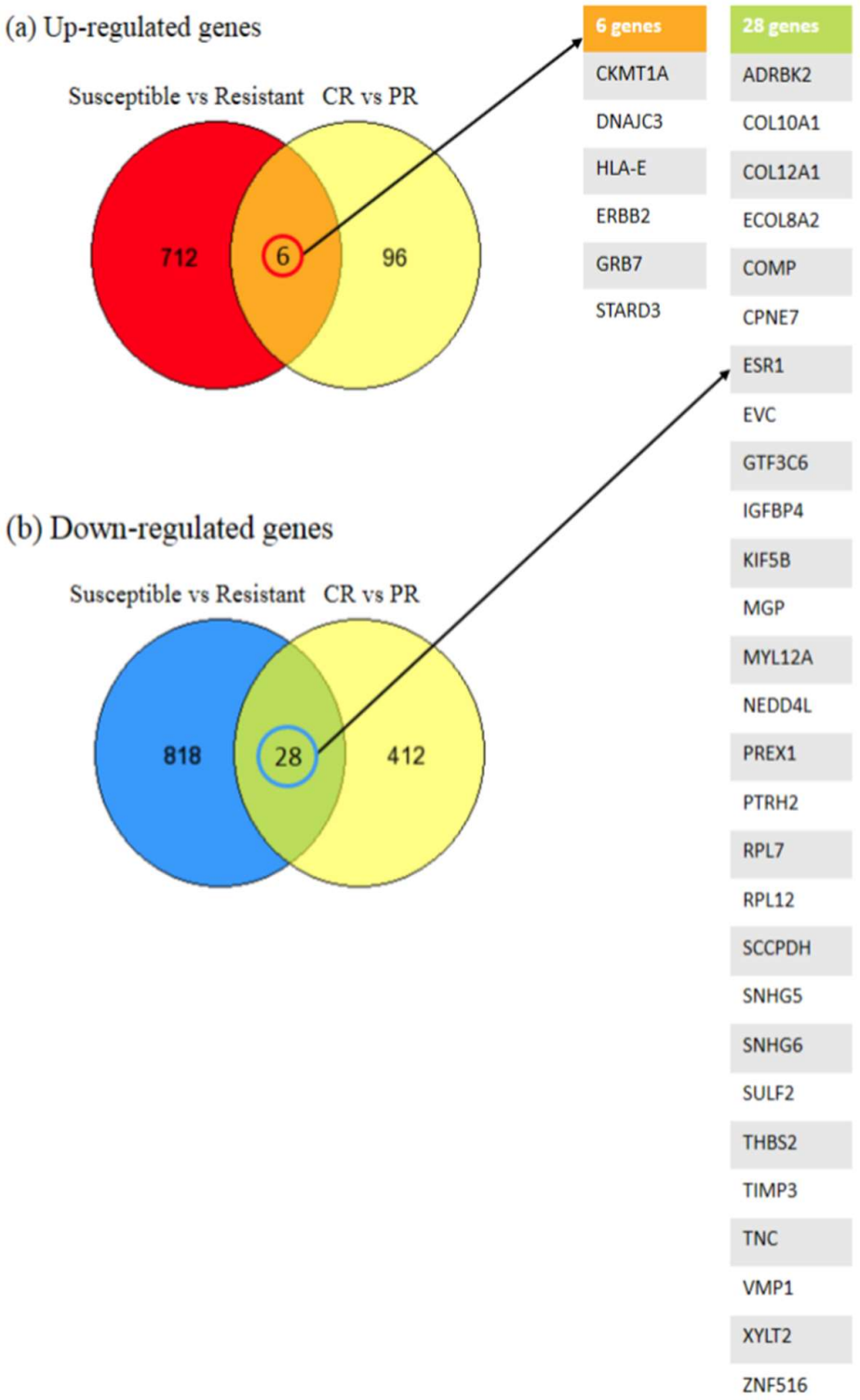

Figure 4. Venn diagram for up and down regulated genes in comparisons of treatment response group and cell lines (Genes are listed in Table 2) 
Table 2. List of upregulated and downregulated genes by comparing treatment response groups and cell lines

\begin{tabular}{|c|c|c|c|}
\hline $\begin{array}{c}\text { Official } \\
\text { gene } \\
\text { symbol }\end{array}$ & Gene name & Fold change & $p$-value \\
\hline & & & up regulated \\
\hline CKMT1A & Creatine kinase, mitochondrial 1A & 2.705126 & $3.58348 \mathrm{E}-10$ \\
\hline DNAJC3 & $\begin{array}{l}\text { DnaJ heat shock protein family (Hsp40) member } \\
\mathrm{C3}\end{array}$ & 3.444459 & 8.97839E-15 \\
\hline$H L A-E$ & Major histocompatibility complex, class I, E & 2.617408 & $5.3738 E-09$ \\
\hline$E R B B 2$ & ERB-B2 receptor tyrosine kinase 2 & 2.975533 & 0.038739022 \\
\hline GRB 7 & Growth factor receptor-bound protein 7 & 3.446123 & 0.043778814 \\
\hline STARD3 & StAR-related lipid transfer domain-containing 3 & 2.646363 & 0.038739022 \\
\hline \multicolumn{4}{|c|}{ Downregulated } \\
\hline ADRBK2 & Adrenergic, beta, receptor kinase 2 & -2.381299 & 0.000262452 \\
\hline COL10A1 & Collagen type $X$ alpha 1 chain & -2.842246 & 0.003024269 \\
\hline COL12A1 & Collagen type XII alpha 1 chain & -2.578220 & 0.003024269 \\
\hline COL8A2 & Collagen type VIII alpha 2 chain & -2.946618 & 0.003024269 \\
\hline СОМР & Cartilage oligomeric matrix protein & -2.334411 & 0.003024269 \\
\hline CPNE7 & Copine 7 & -4.318968 & 0.001835519 \\
\hline ESR1 & Estrogen receptor 1 & -3.228467 & 0.000250757 \\
\hline$E V C$ & EVC ciliary complex subunit 1 & -2.167110 & 0.02418241 \\
\hline GTF3C6 & General transcription factor IIIC subunit 6 & -4.740366 & $1.45291 \mathrm{E}-40$ \\
\hline IGFBP4 & Insulin-like growth factor-binding protein 4 & -3.693364 & 0.000206995 \\
\hline KIF5B & Kinesin family member 5B & -2.191572 & 0.000860611 \\
\hline MGP & Matrix Gla protein & -6.096913 & 0.02418241 \\
\hline MYL12A & Myosin light chain 12A & -3.147437 & 0.018231381 \\
\hline NEDD4L & $\begin{array}{l}\text { Neural precursor cell expressed, developmentally } \\
\text { downregulated 4-like, E3 ubiquitin protein ligase }\end{array}$ & -2.843762 & 0.005725031 \\
\hline PREXI & $\begin{array}{l}\text { Phosphatidylinositol-3,4,5-trisphosphate- } \\
\text { dependent Rac exchange factor } 1\end{array}$ & -2.574266 & 0.018231381 \\
\hline PTRH2 & Peptidyl-tRNA hydrolase 2 & -5.670545 & 0.001051079 \\
\hline RPL7 & Ribosomal protein $\mathrm{L} 7$ & -4.210654 & 0.000440835 \\
\hline RPL12 & Ribosomal protein L12 & -2.658676 & 0.000155619 \\
\hline SCCPDH & Saccharopine dehydrogenase (putative) & -2.520526 & 0.000302021 \\
\hline SNHG5 & Small nucleolar RNA host gene 5 & -3.406315 & 0.004434402 \\
\hline SNHG6 & Small nucleolar RNA host gene 6 & -3.584739 & 0.000797883 \\
\hline SULF2 & Sulfatase 2 & -3.678365 & 0.02418241 \\
\hline THBS2 & Thrombospondin 2 & -3.236972 & 0.001835519 \\
\hline TIMP3 & TIMP metallopeptidase inhibitor 3 & -2.256933 & 0.017523049 \\
\hline TNC & Tenascin C & -3.933920 & 0.001179376 \\
\hline VMP1 & Vacuole membrane protein 1 & -2.274594 & 0.001835519 \\
\hline XYLT2 & Xylosyltransferase 2 & -3.793383 & 0.00471615 \\
\hline ZNF516 & Zinc finger protein 516 & -2.193201 & 0.001835519 \\
\hline
\end{tabular}




\section{Discussion}

We found that expression changes in 34 genes in several pathways were related to the response to trastuzumabcontaining treatment in HER2-type breast cancer, interfered with adhesion to other cells or tissues (focal adhesion), and regulated ECM-receptor interactions and phagosome action. As a result, the decreased tumor invasiveness and enhanced drug effects might be considered as a potential mechanism for better drug response in the CR group. Of note, functional pathway analysis showed that these pathways affect the HER2 response by interacting with the PI3K pathway and the MAP kinase pathway. As previously reported by Toomey et al. and other investigators, high activation of the PI3K pathway is associated with poor response to anti-HER2 therapy in HER2-positive breast cancer patients.[12, 13]

Neoadjuvant chemotherapy (NAC) is utilized in the context of locally advanced breast cancer to downstage tumors, improve operability, and increase the chances of breast-conserving surgery.[14] Patients receiving NAC share equivalent disease progression and overall survival with those receiving postoperative chemotherapy only.[15] Currently, trastuzumab is the HER2-targeted therapy approved by the United States Food and Drug Administration for HER2-positive breast cancer treatment in neoadjuvant, adjuvant, and metastatic settings. Since 2013, Korean health insurance coverage for trastuzumab has expanded to the neoadjuvant setting; hence, the number of patients who can undergo this treatment has increased. Because neoadjuvant therapy can be administered only once before surgery, prediction of the therapeutic response is important.

Trastuzumab imparts anticancer effects via various mechanisms, one of which is the immunological mechanism of NK cell-mediated cancer cell killing by inducing antibody-dependent cell-mediated cytotoxicity (ADCC). The degree of T-cell infiltration at diagnosis has been found to be correlated with post-treatment response and pCR induction. In this study, we showed that the overexpression of HLA-E correlated with pCR. HLA-E is one of the non-classical HLA-I molecules that plays a key role in the immune surveillance of NK cells and is known to determine tumor-immune interaction.[16] The overexpression of HLA-E suggests that trastuzumab might intensify ADCC. Thus, future research investigating the potential interaction between HLA-E expression and increase in immune cell activity after chemotherapy might validate our hypothesis.

Attainment of pCR with neoadjuvant anti-HER2 targeted chemotherapy is a crude surrogate for long-term survival in HER2-positive breast cancer patients. Hou et al. demonstrated that HER2 intratumoral heterogeneity is associated with incomplete response to anti-HER2 neoadjuvant chemotherapy.[17] Our study suggested that genes that are unique to HER2 and related to this HER2 intratumoral heterogeneity are associated with the functional pathways for cell signaling, such as the cell cycle and cell structure. Nahta et al. reported that cell signaling pathways were related to a mechanism underlying resistance to HER2-targeted therapy in human breast cancer[4] This can be inferred from the fact that tumor cells continue to undergo mitosis, and that there is a greater chance of resistance to trastuzumab during this process. With this strategy, our sub-pathway identification approach is highly effective for discovering accurate pathological biomarkers and pathways. The pathways related to the cell cycle or cell activity are strongly associated with drug response. Rios-Luci et al. performed mRNA microarrays using HER2-resistant cell lines and conducted differential gene expression and 
pathway analysis to identify mechanisms underlying intrinsic and acquired resistance to T-DM1.[18] They also suggested that the different anti-HER2 therapy response mechanism might be related to the functional pathway for cell growth associated with cell structure. According to these results, if poor drug response is initially suspected in patients with HER2-positive breast cancer, treatment with cancer-susceptible drugs or surgery may be used to reduce tumor burden, reduce the final failure rate, and improve the disease prognosis.

The lack of the DR group in this experiment could be considered a limitation of the present study. Tissues from SD or PD groups could have helped predict more diverse responses. Furthermore, the inability to control the hormone receptor status in the tumor tissue could be a confounding factor. However, the treatment response was judged immediately after NAC of the surgical tissue with trastuzumab, which was applied to all patients with the same chemotherapy regimen; hence, this was not a drawback of the study.

The second limitation was the relatively low number of enrolled subjects, although the patients had been treated for 10 years. Of all the patients with HER2-positive breast cancer, only 25-30\% underwent neoadjuvant systemic therapy with trastuzumab before surgery.[19] Some patients refused to give their tumor tissue samples for research purposes. Some patients were referred to our institution with preoperative biopsy results from other hospitals and received cancer treatment without re-biopsy. Therefore, it was difficult to obtain a sufficient number of fresh frozen surgical tissues and paraffin-embedded preoperative biopsy tissues, and the final number of tissues that could be used in the experiments was small.

To overcome these drawbacks, we designed additional experiments for validation using trastuzumabsusceptible and trastuzumab-resistant cell lines. We have already used a similar validation method in the analysis of metastatic HER2-positive breast cancers in an earlier study,[20] which successfully proved its validity. Therefore, it could be effective in yielding favorable results in this experiment.

At the beginning of this study, we chose to use the GeneChip microarray and big data analysis as the study method, expecting to obtain accurate results by these popular research tools. This technology is effective for identifying global changes in cancer incidence and development. The application of gene expression profiling is an excellent approach to integrate multiple molecular phenomena and mechanisms underlying cancer development. Tumorigenesis is accompanied by the destruction of various cell pathways, including the cell cycle and cell growth, survival, and apoptosis. Gene expression microarrays provide detailed explanations of molecular fingerprinting of tumors by simultaneously analyzing thousands of genes in specific tumors. While several studies using these methods have been published,[21-23] more rapid technological progress has continued.

Although our study did not suggest a specific gene as a biomarker immediately applicable to clinical practice, we could reveal some potential genes and pathways that should be studied more extensively. Studies to identify practical prognostic markers for trastuzumab treatment response are essential.

In future studies, quantitative RNAseq experiments might assess the difference in gene expression among diff erent samples more accurately. Ultimately, by balancing the estimated risks and benefits, a personalized appro 
ach can be achieved on the basis of an algorithmic approach.

\section{Conclusions}

In our study, 34 genes that are mainly expressed in the several cell signaling pathways closely linked to the $\mathrm{H}$ ER2 expression pathway were found to be related to the drug response to antiHER2 therapy by regulating the cell cycle. This multigene assaybased study of related pathways and identified genes might act as the cornerstone for a greater insight into bre ast cancer signal transduction and the possible prediction of treatment response to targeted therapies such as tr astuzumab.

Author Contributions: You, Lee, DH Kim and Bae conceived and designed the study. You, Park and KT Kim wrote the paper; Nam and You designed the figures; Nam, Kwon and You summarised the data, analysed the data and created the tables; Lee, KT Kim and Bae reviewed and edited the manuscript. All authors read, critically reviewed and approved the final manuscript.

Funding: This research was supported by the Eulji Medi-Bio Research Institute Grant 2016-SN0002.

Acknowledgments: In this section you can acknowledge any support given which is not covered by the author contribution or funding sections. This may include administrative and technical support, or donations in kind (e.g., materials used for experiments).

Conflicts of Interest: The authors declare no conflict of interest.The funders had no role in the design of the study; in the collection, analyses, or interpretation of data; in the writing of the manuscript, or in the decision to publish the results.

\section{References}

1. Chang HR. Trastuzumab-based neoadjuvant therapy in patients with HER2-positive breast cancer. Cancer. 2010;116(12):2856-67. doi:10.1002/cncr.25120.

2. Vu T, Claret FX. Trastuzumab: updated mechanisms of action and resistance in breast cancer. Front Oncol. 2012;2:62. doi:10.3389/fonc.2012.00062.

3. Carter P, Presta L, Gorman CM, Ridgway JB, Henner D, Wong WL et al. Humanization of an anti-p185HER2 antibody for human cancer therapy. Proc Natl Acad Sci U S A. 1992;89(10):4285-9.

4. Nahta R, Yu D, Hung MC, Hortobagyi GN, Esteva FJ. Mechanisms of disease: understanding resistance to HER2-targeted therapy in human breast cancer. Nat Clin Pract Oncol. 2006;3(5):269-80 . doi:10.1038/ncponc0509.

5. Gianni L, Eiermann W, Semiglazov V, Manikhas A, Lluch A, Tjulandin S et al. Neoadjuvant chemotherapy with trastuzumab followed by adjuvant trastuzumab versus neoadjuvant chemotherapy alone, in patients with HER2-positive locally advanced breast cancer (the NOAH trial): a randomised controlled superiority trial with a parallel HER2-negative cohort. Lancet. 2010;375(9712):377-84. doi:10.1016/S0140-6736(09)61964-4.

6. Valachis A, Mauri D, Polyzos NP, Chlouverakis G, Mavroudis D, Georgoulias V. Trastuzumab combined to neoadjuvant chemotherapy in patients with HER2-positive breast cancer: a systematic review and meta-analysis. Breast. 2011;20(6):485-90. doi:10.1016/j.breast.2011.06.009.

7. Symmans WF, Peintinger F, Hatzis C, Rajan R, Kuerer H, Valero V et al. Measurement of residual breast cancer burden to predict survival after neoadjuvant chemotherapy. J Clin Oncol. 2007;25(28):4414-22. doi:10.1200/JCO.2007.10.6823. 
8. Roukos DH. Beyond HER2 and trastuzumab: heterogeneity, systems biology, and cancer origin research may guide the future for personalized treatment of very early but aggressive breast cancer. J Clin Oncol. 2010;28(17):e279-80; author reply e82-3. doi:10.1200/JCO.2009.27.7061.

9. von der Heyde S, Wagner S, Czerny A, Nietert M, Ludewig F, Salinas-Riester G et al. mRNA profiling reveals determinants of trastuzumab efficiency in HER2-positive breast cancer. PLoS One. 2015;10(2):e0117818. doi:10.1371/journal.pone.0117818.

10. G DIC, Marengo G, Albanese NN, Marabeti MR, Musso R, Cancemi P et al. Proteomic profiling of Trastuzumab (Herceptin(R))-sensitive and -resistant SKBR-3 breast cancer cells. Anticancer Res. 2013;33(2):489-503.

11. Huang DW, Sherman BT, Tan Q, Collins JR, Alvord WG, Roayaei J et al. The DAVID Gene Functional Classification Tool: a novel biological module-centric algorithm to functionally analyze large gene lists. Genome Biol. 2007;8(9):R183. doi:10.1186/gb-2007-8-9-r183.

12. Loibl S, von Minckwitz G, Schneeweiss A, Paepke S, Lehmann A, Rezai M et al. PIK3CA mutations are associated with lower rates of pathologic complete response to anti-human epidermal growth factor receptor 2 (her2) therapy in primary HER2-overexpressing breast cancer. J Clin Oncol. 2014;32(29):3212-20. doi:10.1200/JCO.2014.55.7876.

13. Toomey S, Eustace AJ, Fay J, Sheehan KM, Carr A, Milewska M et al. Impact of somatic PI3K pathway and ERBB family mutations on pathological complete response (pCR) in HER2-positive breast cancer patients who received neoadjuvant HER2-targeted therapies. Breast Cancer Res. 2017;19(1):87. doi:10.1186/s13058017-0883-9.

14. Kaufmann M, von Minckwitz G, Smith R, Valero V, Gianni L, Eiermann W et al. International expert panel on the use of primary (preoperative) systemic treatment of operable breast cancer: review and recommendations. J Clin Oncol. 2003;21(13):2600-8. doi:10.1200/JCO.2003.01.136.

15. Mauri D, Pavlidis N, Ioannidis JP. Neoadjuvant versus adjuvant systemic treatment in breast cancer: a metaanalysis. J Natl Cancer Inst. 2005;97(3):188-94. doi:10.1093/jnci/dji021.

16. Algarra I, Garcia-Lora A, Cabrera T, Ruiz-Cabello F, Garrido F. The selection of tumor variants with altered expression of classical and nonclassical MHC class I molecules: implications for tumor immune escape. Cancer Immunol Immunother. 2004;53(10):904-10. doi:10.1007/s00262-004-0517-9.

17. Hou Y, Nitta H, Wei L, Banks PM, Portier B, Parwani AV et al. HER2 intratumoral heterogeneity is independently associated with incomplete response to anti-HER2 neoadjuvant chemotherapy in HER2-positive breast carcinoma. Breast Cancer Res Treat. 2017;166(2):447-57. doi:10.1007/s10549-017-4453-8.

18. Rios-Luci C, Garcia-Alonso S, Diaz-Rodriguez E, Nadal-Serrano M, Arribas J, Ocana A et al. Resistance to the Antibody-Drug Conjugate T-DM1 Is Based in a Reduction in Lysosomal Proteolytic Activity. Cancer Res. 2017;77(17):4639-51. doi:10.1158/0008-5472.CAN-16-3127.

19. Shinde AM, Zhai J, Yu KW, Frankel P, Yim JH, Luu T et al. Pathologic complete response rates in triplenegative, HER2-positive, and hormone receptor-positive breast cancers after anthracycline-free neoadjuvant chemotherapy with carboplatin and paclitaxel with or without trastuzumab. Breast. 2015;24(1):18-23. doi:10.1016/j.breast.2014.10.008.

20. Nam S, Chang HR, Jung HR, Gim Y, Kim NY, Grailhe R et al. A pathway-based approach for identifying biomarkers of tumor progression to trastuzumab-resistant breast cancer. Cancer Lett. 2015;356(2 Pt B):880-90. doi:10.1016/j.canlet.2014.10.038. 
21. Berns K, Horlings HM, Hennessy BT, Madiredjo M, Hijmans EM, Beelen K et al. A functional genetic approach identifies the PI3K pathway as a major determinant of trastuzumab resistance in breast cancer. Cancer Cell. 2007;12(4):395-402. doi:10.1016/j.ccr.2007.08.030.

22. Makoukji J, Makhoul NJ, Khalil M, El-Sitt S, Aldin ES, Jabbour M et al. Gene expression profiling of breast cancer in Lebanese women. Sci Rep. 2016;6:36639. doi:10.1038/srep36639.

23. Llombart-Cussac A, Cortes J, Pare L, Galvan P, Bermejo B, Martinez N et al. HER2-enriched subtype as a predictor of pathological complete response following trastuzumab and lapatinib without chemotherapy in early-stage HER2-positive breast cancer (PAMELA): an open-label, single-group, multicentre, phase 2 trial. Lancet Oncol. 2017;18(4):545-54. doi:10.1016/S1470-2045(17)30021-9. 